\title{
Synthesis of Porphyrins from $\alpha$-Unsubstituted Dipyrromethanes
}

\author{
T. V. Lyubimova, ${ }^{\mathrm{a}, \mathrm{b}}$ S. A. Syrbu, ${ }^{\mathrm{a}}$ and A. S. Semeikin ${ }^{\mathrm{a}}$ \\ Dedicated to Academician of Russian Academy of Sciences O. G. Sinyashin \\ on the occasion of his $60^{\text {th }}$ birthday
}

${ }^{a}$ Ivanovo State University of Chemistry and Technology, 15300 Ivanovo, Russian Federation

${ }^{\mathrm{b}}$ G.A. Krestov Institute of Solution Chemistry, Russian Academy of Sciences, 153045 Ivanovo, Russian Federation

@Corresponding authorE-mail:semeikin@isuct.ru

\begin{abstract}
The conditions for the porphyrin synthesis by condensation of $\alpha$-unsubstituted dipyrromethanes and ortho-formic ester in the presence of organic acids are investigated. The optimal reaction conditions are found, and a series of $\beta$ and meso-substituted porphyrins is synthesized. The methods of the $\alpha$-unsubstituted dipyrromethanes synthesis from pyrroles obtained by Knorr method are designed. The synthesized compounds were characterized by spectral methods.
\end{abstract}

Keywords: Synthesis, dipyrromethanes, porphyrins.

\section{Синтез порфиринов из $\alpha$-незамещенных дипироллилметанов}

\author{
Т. В. Аюбимова, ${ }^{\mathrm{a}, \mathrm{b}}$ С. А. Сырбу, А. С. Семейкин ${ }^{\mathrm{a}}$ \\ Посвящается Акаgемику РАН О.Г. Синяшину по случаю его 60-летнего юбилея
}

\author{
а Ивановский государственный химико-технологический университет, 153000 Иваново, Россия \\ ${ }^{\mathrm{b}}$ Институт химии растворов им. Г.А. Крестова РАН, 153045 Иваново, Россия \\ @E-mail: semeikin@isuct.ru
}

\begin{abstract}
Изучень условия синтеза порфиринов конденсацией $\alpha$-незамещзенных дипирролилметанов с ортомуравьиным эфиромв присутствии органических кислот. Найдены оптимальныеусловия проведения реакиии, исинтезирован ряд $\beta$ - и мезо-замещенных порфиринов. Разработаны методы синтеза а-незамещенных дипирролилметанов из пирролов, полученных по методу Кнорра. Синтезированные соединения охарактеризованы спектральными методами.
\end{abstract}

Ключевые слова: Синтез, дипирролилметаны, порфирины.

Tetrapyrrolic macroheterocycles have found widespread application as new functional materials with useful properties. In particular, synthetic porphyrins and their metal complexes are widely used as model compounds for studies of biochemical processes, catalysts and medicinal drugs. ${ }^{[1]}$ Easily available meso-tetraarylporphyrins $\mathbf{1}$ and more related to natural $\beta$-octaalkylporphyrins $\mathbf{2}$ are the most common in practice. Some of them, like 5,10,15,20-tetraphenylporphyrin $1(\mathrm{Ar}=\mathrm{Ph})$ and 2,3,7,8,12,13,17,18-octaethylporphyrin 2 $(\mathrm{Alk}=\mathrm{Et})$ have symmetrically arranged substituents and are sufficiently soluble in organic solvents, unlike a more simple 2,3,7,8,12,13,17,18-octamethyporphyrin $2(\mathrm{Alk}=\mathrm{Me})$ which is almost not soluble in the majority of organic solvents.

Of interest is a simple method of synthesis of $\beta$-substituted alkylporphyrins with the general structure of 6 by condensation of $\alpha$-carboxydipyrromethanes 3 $(\mathrm{X}=\mathrm{COOH})$ with triethyl-ortho-formate in the presence of an acid (Scheme 1)..$^{[2-7]}$

This reaction is assumed to proceed via decarboxylation of the original dipyrromethane 3 with the subsequent formylation to give an $\alpha$-formyl derivative 4 which undergoes cyclocondensation unto porphodimethene $\mathbf{5}$ with the 


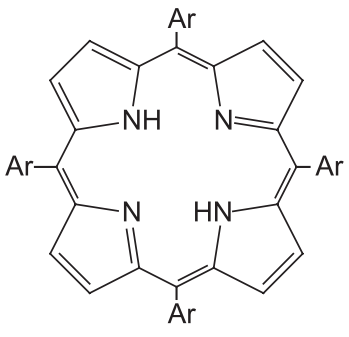

1

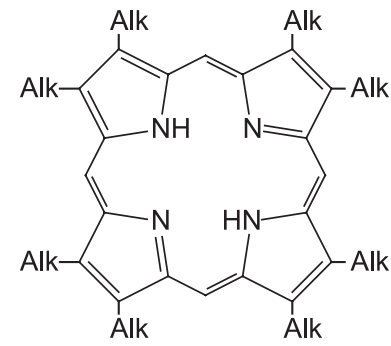

2 further oxidation into porphyrin $\mathbf{6}$. To achieve this transformation, trichloroacetic or trifluoroacetic acid is used as the catalyst, ${ }^{[2-7]}$ the acid and ortho-formate are used in significant excess with respect to dipyrromethane and the reaction is carried out in chloroform or methylene chloride in air at reflux.

Finding the optimal conditions for this reaction was the goal of the present study.

An improved method was developed for the synthesis of $\alpha$-unsubstituted dipyrromethanes 3 and 11 (Scheme 2) from relatively easily available pyrroles $7 \cdot{ }^{[8]}$ For the synthesis of symmetrically meso-unsubstituted dipyrromethanes $\mathbf{1 1}$ radical bromination of pyrrolic $\alpha$-methyl group in compounds 7 was performed to afford $\alpha$-bromomethylpirroles 8 , in which under the reaction conditions bromine undergoes nucleophilic substitution with the formation of acetoxymethylpyrroles 9 that without preliminary purification undergo self-condensation to 5,5'-diethoxycarbonyldipyrromethanes 10. Decarboxylation of the latter by potassium hydroxide in refluxing ethylene glycol leads to the target compounds $\mathbf{1 1}$.

meso-Substituted dipyrromethanes $\mathbf{3}$ are also synthesized from pyrroles 7 by radical chlorination with sulfuryl chloride in ether with the formation of $\alpha$-trichloromethylpyrroles 12 followed by their hydrolysis into $\alpha$-carboxypyrroles 13, decarboxylation of the latter to $\alpha$-unsubstituted pyrroles 15 (upon heating or by reduction of the intermediate $\alpha$-iodopyrroles 14). By the condensation of 15 with aldehydes meso-substituted 5,5'-diethoxycarbonyldipyrromethanes $\mathbf{1 6}$ were obtained and decarboxylated to afford 3 .

As a model reaction we chose the synthesis of 2,8,12,18-tetramethyl-3,7,13,17-tetra- $n$-amylporphyrin, which is well-soluble in most common solvents and can be easily isolated from the reaction mixture by column chromatography.

We have found it was more beneficial to use $\alpha$-unsubstituted dipyrromethane $\mathbf{1 1}$ for the synthesis, not its $\alpha$-carboxyl derivative, because of the yield in this case was significantly increased (Table 1) (exp. 1,2). The concentration of dipyrromethane affects the porphyrin yield weakly (exp. $3,6)$. Using other non-polar solvents instead of chloroform slightly lowers the yield (exp. 7-9), with the lowest one being in the case of methylene chloride (exp. 9) which is possibly caused by its low boiling point. A two-fold increase of the concentration of ortho-formate and trifluoroacetic acid with respect to dipyrromethane (from 3-fold excess to 6-fold) leads to a decrease of the porphyrin yield by $10 \%$, while reduction of their content to 1.5 -fold excess gives a boost of the yield by $30 \%$ (exp. 2-4). The oxidant used in the reaction does not influence the yield much (exp. 10-12), however it is lower than for $p$-chloranil (exp. 6) and a two-fold reduction the oxidant amount results in a two-fold reduction of the yield (exp. 13). It is interesting that using atmospheric oxygen as the oxidant leads to an increased yield of the porphyrin (exp. 14). Replacing trifluoroacetic acid with trichloroacetic (exp. 15) leads to a significant drop of the yield. When the reaction time is shortened from 4 to 2 hours the yield is decreased by $14 \%$ (exp. 5).

We used the found optimal reaction conditions to conduct syntheses of a number of porphyrins with substituents of different length at $\beta$-positions of the porphyrin core and with substituents in meso-positions (Table 2). It was established that the porphyrin with only 4 substituents in $\beta$-positions $\left(6, \mathrm{R}, \mathrm{R}^{1}=\mathrm{H}\right)$ is formed with the lowest yield, which is typical even for other porphyrins with unsubstituted $\beta$-positions..$^{[9,10]}$ The porphyrins which are poorly soluble in the majority of organic solvents $(\mathbf{6}$, $\mathrm{R}=\mathrm{Me}, \mathrm{R}^{1}=\mathrm{H} ; \mathbf{6}, \mathrm{R}=\mathrm{Et}, \mathrm{R}^{1}=\mathrm{H} ; \mathbf{6}, \mathrm{R}=\mathrm{Bz}, \mathrm{R}^{1}=\mathrm{H}$, and $\mathbf{6}$, $\mathrm{R}=\mathrm{Et}, \mathrm{R}^{1}=\mathrm{Ph}$ ) are also formed with low yields and need to be purified by re-precipitation with trifluoroacetic acid from the reaction mixture. For porphyrin $6\left(R=A m, R^{1}=H\right)$ as well as for the other porphyrins the yields are much higher when an unsubstituted dipyrromethane is used instead of

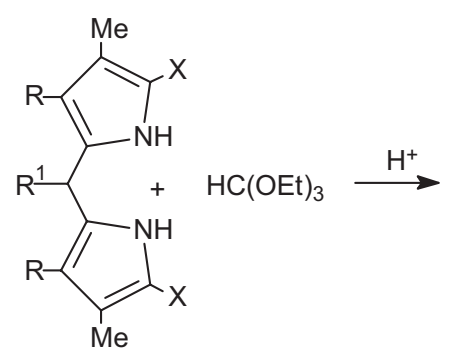

3

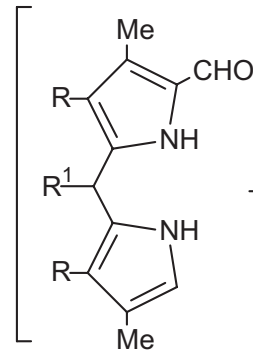

4

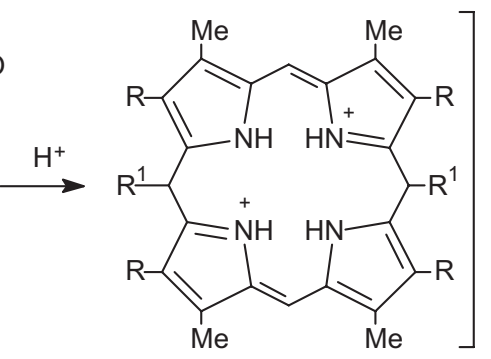

5

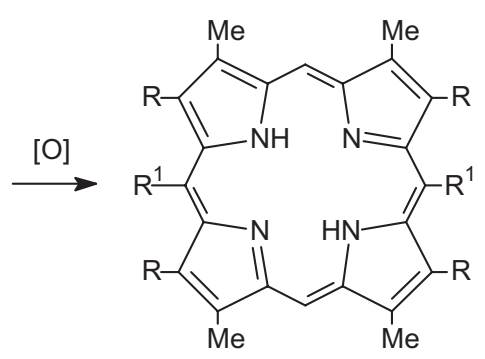

6

Scheme 1. 


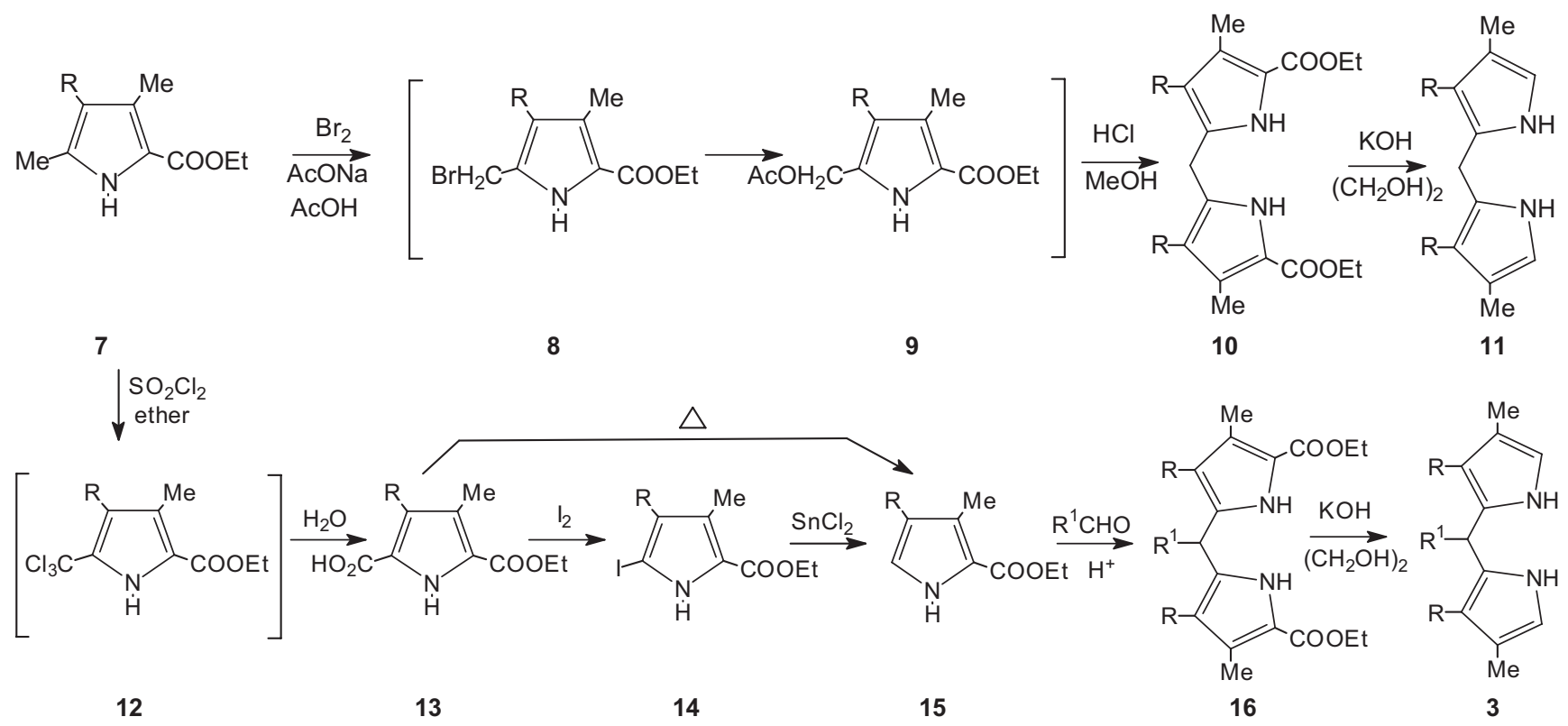

Scheme 2.

Table 1. Examples of the synthesis of 2,8,12,18-tetramethyl-3,7,13,17-tetra- $n$-amylporphyrin $6\left(\mathrm{R}=\mathrm{Am}, \mathrm{R}^{1}=\mathrm{H}\right)$ by the condensation of dipyrromethane $11(\mathrm{R}=\mathrm{Am})$ with ethyl ortho-formate in the presence of an acid $(\mathrm{CA}=$ chloranil, $\mathrm{BQ}=$ benzoquinone $)$.

\begin{tabular}{cccccccc}
\hline $\mathrm{N}$ & $\mathbf{3}(\mathrm{mmol})$ & $\begin{array}{c}\text { Solvent } \\
(\mathrm{ml})\end{array}$ & $\begin{array}{c}\mathrm{HC}(\mathrm{OEt})_{3} \\
\text { (equivalents } \\
\text { against 3) }\end{array}$ & $\begin{array}{c}\mathrm{CF}_{3} \mathrm{COOH} \\
\text { (equivalents } \\
\text { against 3) }\end{array}$ & $\begin{array}{c}\text { Oxidant } \\
\text { (equivalents against 3) }\end{array}$ & Time (h) & $\begin{array}{c}\text { Yield } \\
(\%)\end{array}$ \\
\hline 1 & $2.0^{\mathrm{a}}$ & $100\left(\mathrm{CHCl}_{3}\right)$ & 6.3 & 7.1 & $1.0(\mathrm{CA})$ & 4 & 39.4 \\
2 & 1.91 & $100\left(\mathrm{CHCl}_{3}\right)$ & 6.3 & 7.1 & $1.0(\mathrm{CA})$ & 4 & 68.0 \\
3 & 1.91 & $100\left(\mathrm{CHCl}_{3}\right)$ & 3.1 & 3.6 & $1.0(\mathrm{CA})$ & 4 & 83.7 \\
4 & 1.91 & $100\left(\mathrm{CHCl}_{3}\right)$ & 1.6 & 1.8 & $1.0(\mathrm{CA})$ & 4 & 53.1 \\
5 & 1.91 & $100\left(\mathrm{CHCl}_{3}\right)$ & 3.1 & 3.6 & $1.0(\mathrm{CA})$ & 2 & 69.2 \\
6 & 1.84 & $50\left(\mathrm{CHCl}_{3}\right)$ & 3.3 & 3.7 & $1.0(\mathrm{CA})$ & 4 & 80.6 \\
7 & 1.97 & $50\left(\mathrm{C}_{6} \mathrm{H}_{6}\right)$ & 3.1 & 3.5 & $1.0(\mathrm{CA})$ & 4 & 70.3 \\
8 & 1.97 & $50\left(\mathrm{CCl}_{4}\right)$ & 3.1 & 3.5 & $1.0(\mathrm{CA})$ & 4 & 67.1 \\
9 & 1.97 & $50\left(\mathrm{CH}_{2} \mathrm{Cl}_{2}\right)$ & 3.1 & 3.5 & $1.0(\mathrm{CA})$ & 4 & 58.2 \\
10 & 1.91 & $100\left(\mathrm{CHCl}_{3}\right)$ & 3.1 & 3.6 & $1.0(\mathrm{BQ})$ & 4 & 75.7 \\
11 & 1.97 & $100\left(\mathrm{CHCl}_{3}\right)$ & 3.1 & 3.5 & $1.0\left(\mathrm{I}_{2}\right)$ & 4 & 67.1 \\
12 & 1.97 & $50\left(\mathrm{CHCl}_{3}\right)$ & 3.1 & 3.5 & $1.0(\mathrm{DDQ})$ & 4 & 67.1 \\
13 & 1.84 & $100\left(\mathrm{CHCl}_{3}\right)$ & 3.3 & 3.7 & $0.5(\mathrm{CA})$ & 4 & 40.3 \\
14 & 1.65 & $50\left(\mathrm{CHCl}_{3}\right)$ & 3.6 & 4.1 & Air & 4 & 87.5 \\
15 & 1.59 & $50\left(\mathrm{CHCl}_{3}\right)$ & 3.8 & $4.0\left(\mathrm{Cl}_{3} \mathrm{CCO}_{2} \mathrm{H}\right)$ & $1.0(\mathrm{CA})$ & 4 & 54.1 \\
\hline
\end{tabular}

a dipyrromethane-5,5'-dicarboxylic acid

its $5,5^{\prime}$-carboxyl derivative. The reason for the relatively low yields of porphyrins obtained from meso-substituted dipyrromethanes $\mathbf{3}$ is not clear, it can be possibly related to stable unreactive conformations of the starting compounds in the reaction media which hinders cyclization.

\section{Experimental}

The electron absorption spectra (EAS) were recorded on a SPEC SSP-715 scanning spectrophotometer in chloroform. The ${ }^{1} \mathrm{H}$
NMR spectra were obtained on a Bruker 500 instrument (TMS as the internal standard, $500 \mathrm{MHz}, 20^{\circ} \mathrm{C}$ ) in the shared research center "Upper Volga regional physicochemical research center" of the Krestov Institute of Solution Chemistry of the Russian Academy of Sciences. Thin layer chromatography (TLC) was performed on Silufol plates.

2-Chloromethyl-4-methyl-3,5-diethoxycarbonylpyrrole. Sulfuryl chloride $(28.4 \mathrm{ml}, 0.35 \mathrm{~mol})$ in acetic acid $(60 \mathrm{ml})$ was added to a stirred suspension of 2,4-dimethyl-3,5-diethoxycarbonylpyrrole $(84.0 \mathrm{~g}, 0.35 \mathrm{~mol})$ in glacial acetic acid $(700 \mathrm{ml})$. The temperature during the addition was maintained at $50-60{ }^{\circ} \mathrm{C}$, then it was raised to $70{ }^{\circ} \mathrm{C}$ for $0.5 \mathrm{~h}$. The mixture was slowly cooled, the 
Table 2. The yield of porphyrins 6 obtained by the condensation of dipyrromethanes $\mathbf{3}$ or $\mathbf{1 1}$ with ethyl ortho-formate in the presence of trifluoroacetic acid in chloroform, oxidation with air.

\begin{tabular}{ccc}
\hline \multicolumn{2}{c}{ Porphyrin 6 } & Yield (\%) \\
\cline { 1 - 2 } $\mathrm{R}$ & $\mathrm{R}^{1}$ & 5.2 \\
$\mathrm{H}$ & $\mathrm{H}$ & 31.9 \\
$\mathrm{Me}$ & $\mathrm{H}$ & 65.5 \\
$\mathrm{Et}$ & $\mathrm{H}$ & $52.8(64.5)^{\mathrm{a}}$ \\
$\mathrm{Pr}$ & $\mathrm{H}$ & 68.8 \\
$\mathrm{Bu}$ & $\mathrm{H}$ & $87.5(39.4)$ \\
$\mathrm{Am}$ & $\mathrm{H}$ & $52.5(25.7)$ \\
$\mathrm{Hex}$ & $\mathrm{H}$ & $43.5(25.7)$ \\
$\mathrm{Hep}$ & $\mathrm{H}$ & 31.4 \\
$\mathrm{Bz}$ & $\mathrm{H}$ & 35.4 \\
$\mathrm{Et}$ & $\mathrm{Ph}$ & $25.7(15.5)$ \\
$\mathrm{Et}$ & $4-\mathrm{MeOPh}$ & 35.2 \\
$\mathrm{Bu}$ & $\mathrm{PH}$ & 39.2 \\
$\mathrm{Bu}$ & $4-\mathrm{MeOPh}$ & \\
\hline
\end{tabular}

athe yield for dipyrromethane-5,5'-dicarboxylic acid as the starting material is given in brackets.

pyrrole precipitate filtered, washed with $100 \mathrm{ml}$ of petroleum ether and dried in air at ambient temperature. Yield: $64.6 \mathrm{~g}(67.4 \%)$. M.p.: $153-154{ }^{\circ} \mathrm{C} .{ }^{1} \mathrm{H}$ NMR $\left(\mathrm{CDCl}_{3}\right) \delta_{\mathrm{H}}$ ppm: 9.75 (bs, $\left.1 \mathrm{H}, \mathrm{NH}\right)$; 4.99 (s, 2H, $\left.\mathrm{CH}_{2} \mathrm{Cl}\right) ; 4.40$ (q, 2H, $\left.J=7.1 \mathrm{~Hz}, \mathrm{CH}_{2}-\mathrm{Et}\right) ; 4.33$ (q, 2H, $\left.J=7.1 \mathrm{~Hz}, \mathrm{OCH}_{2}-\mathrm{Et}\right) ; 2.59\left(\mathrm{~s}, 3 \mathrm{H}, \mathrm{CH}_{3}\right) ; 1.41(\mathrm{q}(\mathrm{t}+\mathrm{t}), 6 \mathrm{H}, J=7.1 \mathrm{~Hz}$, $\left.\mathrm{CH}_{3}-\mathrm{Et}\right)$.

3,3',5,5'-Tetraethoxycarbonyl-4,4'-dimethyldipyrromethane (10, $\mathrm{R}=\mathrm{CO}$ Et). 2-Chloromethyl-4-methyl-3,5-diethoxycarbonylpyrrole $(60.0 \mathrm{~g}, 0.22 \mathrm{~mol})$ was dissolved with stirring in refluxing acetic acid $(80 \mathrm{ml})$, then water $(80 \mathrm{ml})$ was gradually added, and the mixture was further refluxed for 1.5 hours, slowly cooled, and the precipitate was filtered, washed with water, dried in air at ambient temperature. Yield: $45.3 \mathrm{~g}(89.0 \%)$. M.p.: $134-136{ }^{\circ} \mathrm{C} .{ }^{1} \mathrm{H}$ NMR $\left(\mathrm{CDCl}_{3}\right) \delta_{\mathrm{H}}$ ppm: $10.14(\mathrm{bs}, 2 \mathrm{H}, \mathrm{NH}) ; 4.51\left(\mathrm{~s}, 2 \mathrm{H}, m s-\mathrm{CH}_{2}\right) ; 4.39$ (q, $4 \mathrm{H}, J=7.1 \mathrm{~Hz}, \mathrm{OCH}_{2}-\mathrm{Et}$ ); 4.31 (q, 4H, $J=7.1 \mathrm{~Hz}, \mathrm{OCH}_{2}-\mathrm{Et}$ ); 2.53 $\left(\mathrm{s}, 6 \mathrm{H}, \mathrm{CH}_{3}\right) ; 1.44$ (t, $\left.6 \mathrm{H}, J=7.1 \mathrm{~Hz}, \mathrm{CH}_{3}-\mathrm{Et}\right) ; 1.37$ (t, $6 \mathrm{H}, J=7.1 \mathrm{~Hz}$, $\left.\mathrm{CH}_{3}-\mathrm{Et}\right)$.

5,5'-Diethoxycarbonyl-3,3'-di-n-amyl-4,4'-dimethyldipyrrolylmethane (10, $R=A m)$. A suspension of 2,4-dimethyl-3- $n$-amyl5-carboethoxypyrrole (100.0 g, $0.42 \mathrm{~mol})$ and anhydrous sodium acetate $(69.0 \mathrm{~g}, 0.84 \mathrm{~mole})$ in acetic acid $(500 \mathrm{ml})$ was stirred with cooling in a cold water bath. Bromine $(21.6 \mathrm{ml}, 0.42 \mathrm{~mol})$ was gradually introduced. The suspension was stirred for 0.5 hours and poured into 3 liters of cold water, the precipitate was filtered, washed with water and refluxed for $3 \mathrm{hrs}$ with a mixture of methanol $(0.5 \mathrm{l})$ and concentrated hydrochloric acid $(10 \mathrm{ml})$. After cooling the mixture was left overnight, the precipitate filtered, washed with water and dried. Yield: $54.5 \mathrm{~g}(56.5 \%)$. M.p.: $125-126^{\circ} \mathrm{C} .{ }^{1} \mathrm{H} \mathrm{NMR}\left(\mathrm{CDCl}_{3}\right) \delta_{\mathrm{H}}$ ppm: 9.08 (bs, 2H, NH); 4.26 (q, 4H, J=7.2 Hz, $\mathrm{OCH}_{2}-\mathrm{Et}$ ); 3.88 (s, $\left.2 \mathrm{H}, m s-\mathrm{CH}_{2}\right) ; 2.39$ (t, $\left.4 \mathrm{H},{ }^{1} J=7.3 \mathrm{~Hz}, \mathrm{CH}_{2}-\mathrm{Am}\right) ; 2.28$ (s, $6 \mathrm{H}, \mathrm{CH}_{3}$ ); 1.40 (qv, $4 \mathrm{H},{ }^{1} J=7.3 \mathrm{~Hz}, \mathrm{CH}_{2}-\mathrm{Am}$ ); 1.32 (t, $6 \mathrm{H}, J=7.2 \mathrm{~Hz}, \mathrm{CH}_{3}$-Et); 1.31 (m, 8H, $\left.\mathrm{CH}_{2}-\mathrm{Am}\right) ; 0.90$ (t, $\left.6 \mathrm{H},{ }^{1} \mathrm{~J}=7.3 \mathrm{~Hz}, \mathrm{CH}_{3}-\mathrm{Am}\right)$.

In a similar way the following compounds were obtained:

5,5'-Diethoxycarbonyl-3,3',4,4'-tetramethyldipyrrolylmethane (10, $R=$ Me). Yield: $52.5 \%$. M.p.: $195-197{ }^{\circ} \mathrm{C}\left(197{ }^{\circ} \mathrm{C}\right.$ - lit.). ${ }^{[1]]}{ }^{1} \mathrm{H}$ NMR $\left(\mathrm{CDCl}_{3}\right) \delta_{\mathrm{H}}$ ppm: 9.03 (bs, 2H, NH); 4.25 (q, 4H, $\left.J=7.1 \mathrm{~Hz}, \mathrm{OCH}_{2}-\mathrm{Et}\right) ; 3.84$ (s, $\left.2 \mathrm{H}, m s-\mathrm{CH}_{2}\right) ; 2.24$ (s, 6H, 4,4'- $\mathrm{CH}_{3}$ ); 1.95 (s, 6H, 3,3'- $\left.-\mathrm{CH}_{3}\right) ; 1.30$ (t, $6 \mathrm{H}, J=7.1 \mathrm{~Hz}, \mathrm{CH}_{3}$-Et).

5,5'-Diethoxycarbonyl-3,3'-diethyl-4,4'-dimethyldipyrromethane $(10, R=E t)$. Yield: $57.6 \%$. M.p.: $130-131^{\circ} \mathrm{C}$. $\left(130{ }^{\circ} \mathrm{C}-\right.$ lit.). ${ }^{[1]]}{ }^{1} \mathrm{H} \mathrm{NMR}\left(\mathrm{CDCl}_{3}\right) \delta_{\mathrm{H}}$ ppm: 9.77 (bs, 2H, NH); 4.27 (q, 4H, $\left.J=7.1 \mathrm{~Hz}, \mathrm{OCH}_{2}-\mathrm{Et}\right) ; 3.94\left(\mathrm{~s}, 2 \mathrm{H}, m s-\mathrm{CH}_{2}\right) ; 2.47$ (q, $4 \mathrm{H},{ }^{1} J=7.6 \mathrm{~Hz}$, $\mathrm{CH}_{2}-3,3^{\prime}$-Et); 2.31 (s, 6H, 4,4'- $\mathrm{CH}_{3}$ ); 1.31 (t, 6H, J=7.1 Hz, $\mathrm{CH}_{3}-$ $\mathrm{Et}) ; 1.07$ (t, $6 \mathrm{H},{ }^{1} \mathrm{~J}=7.6 \mathrm{~Hz}, \mathrm{CH}_{3}-3,3$ '-Et). ${ }^{1} \mathrm{H} \mathrm{NMR}\left(\mathrm{CCl}_{4}\right) \delta_{\mathrm{H}} \mathrm{ppm}$ : 9.83 (bs, 2H, NH); 4.03 (q, 4H, J=7.2 Hz, $\mathrm{OCH}_{2}-\mathrm{Et}$ ); 3.73 (s, $2 \mathrm{H}$, $m s-\mathrm{CH}_{2}$ ); 2.30 (q, $4 \mathrm{H},{ }^{1} J=7.5 \mathrm{~Hz}, \mathrm{CH}_{2}-3,3$ '-Et); 2.17 (s, 6H, 4,4'$\mathrm{CH}_{3}$ ); 1.23 (t, $\left.6 \mathrm{H}, J=7.2 \mathrm{~Hz}, \mathrm{CH}_{3}-\mathrm{Et}\right) ; 1.00$ (t, $6 \mathrm{H},{ }^{1} J=7.5 \mathrm{~Hz}, \mathrm{CH}_{3}-$ 3,3'-Et).

5,5'-Diethoxycarbonyl-3,3'-di-n-propyl-4,4'-dimethyldipyrromethane $(10, R=P r)$. Yield: $51.4 \%$. M.p.: $136-138{ }^{\circ} \mathrm{C}\left(116^{\circ} \mathrm{C}\right.$ - lit.). ${ }^{[11]}{ }^{1} \mathrm{H}$ NMR $\left(\mathrm{CDCl}_{3}\right) \delta_{\mathrm{H}}$ ppm: 9.43 (bs, 2H, NH); 4.25 (q, 4H, $\left.J=7.1 \mathrm{~Hz}, \mathrm{OCH}_{2}-\mathrm{Et}\right) ; 3.90\left(\mathrm{~s}, 2 \mathrm{H}, m s-\mathrm{CH}_{2}\right) ; 2.38\left(\mathrm{t}, 4 \mathrm{H},{ }^{1} J=7.4 \mathrm{~Hz}\right.$, $\mathrm{CH}_{2}-\mathrm{Pr}$ ); 2.28 (s, 6H, 4,4'- $\left.\mathrm{CH}_{3}\right) ; 1.46$ (sc, $4 \mathrm{H},{ }^{1} J=7.4 \mathrm{~Hz}, \mathrm{CH}_{2}-\mathrm{Pr}$ ); 1.30 (t, $\left.6 \mathrm{H}, J=7.1 \mathrm{~Hz}, \mathrm{CH}_{3}-\mathrm{Et}\right) ; 0.94$ (t, $\left.6 \mathrm{H},{ }^{1} J=7.4 \mathrm{~Hz}, \mathrm{CH}_{3}-\mathrm{Pr}\right)$.

5,5'-Diethoxycarbonyl-3,3'-di-n-butyl-4,4'-dimethyldipyrromethane (10, $R=B u)$. Yield: $56.0 \%$. M.p.: $118-121{ }^{\circ} \mathrm{C}\left(125{ }^{\circ} \mathrm{C}-\right.$ lit.). ${ }^{[1] ~}{ }^{1} \mathrm{H} \mathrm{NMR}\left(\mathrm{CDCl}_{3}\right) \delta_{\mathrm{H}}$ ppm: 9.25 (bs, 2H, NH); 4.16 (q, 4H, $\left.J=7.1 \mathrm{~Hz}, \mathrm{OCH}_{2}-\mathrm{Et}\right) ; 3.80$ (s, $\left.2 \mathrm{H}, m s-\mathrm{CH}_{2}\right) ; 2.31$ (t, $4 \mathrm{H},{ }^{1} J=7.2 \mathrm{~Hz}$, $\left.\mathrm{CH}_{2}-\mathrm{Bu}\right) ; 2.19$ (s, 6H, 4,4'- $\left.\mathrm{CH}_{3}\right) ; 1.21\left(\mathrm{~m}, 8 \mathrm{H}, \mathrm{CH}_{2}-\mathrm{Bu}\right) ; 1.21$ (t, $\left.6 \mathrm{H}, J=7.1 \mathrm{~Hz}, \mathrm{CH}_{3}-\mathrm{Et}\right) ; 0.84\left(\mathrm{t}, 6 \mathrm{H},{ }^{1} J=7.2 \mathrm{~Hz}, \mathrm{CH}_{3}-\mathrm{Bu}\right) .{ }^{1} \mathrm{H} \mathrm{NMR}$ $\left(\mathrm{CCl}_{4}\right) \delta_{\mathrm{H}} \mathrm{ppm}: 10.09(\mathrm{bs}, 2 \mathrm{H}, \mathrm{NH}) ; 4.15\left(\mathrm{q}, 4 \mathrm{H}, J=7.1 \mathrm{~Hz}, \mathrm{OCH}_{2}-\right.$ $\mathrm{Et}) ; 3.84(\mathrm{~s}, 2 \mathrm{H}, m s-\mathrm{H}) ; 2.34\left(\mathrm{t}, 4 \mathrm{H},{ }^{1} J=7.0 \mathrm{~Hz}, \mathrm{CH}_{2}-\mathrm{Bu}\right) ; 2.18(\mathrm{~s}$, $\left.6 \mathrm{H}, \mathrm{CH}_{3}\right) ; 1.29-1.37\left(\mathrm{~m}, 8 \mathrm{H}, \mathrm{CH}_{2}-\mathrm{Bu}\right) ; 1.26\left(\mathrm{t}, 6 \mathrm{H}, J=7.1 \mathrm{~Hz}, \mathrm{CH}_{3}\right.$ Et); 0.91 (t, $6 \mathrm{H},{ }^{1} J=7.0 \mathrm{~Hz}, \mathrm{CH}_{3}-\mathrm{Bu}$ ).

5,5'-Diethoxycarbonyl-3,3'-di-n-hexyl-4,4'-dimethyldipyrromethane (10, $R=H e x)$. Yield: $62.1 \%$. M.p.: $134-136{ }^{\circ} \mathrm{C}\left(136{ }^{\circ} \mathrm{C}\right.$ - lit.). ${ }^{[1]]}{ }^{1} \mathrm{H}$ NMR $\left(\mathrm{CCl}_{4}\right) \delta_{\mathrm{H}}$ ppm: 9.91 (bs, 2H, NH); 4.16 (q, 4H, $\left.J=7.1 \mathrm{~Hz}, \mathrm{OCH}_{2}-\mathrm{Et}\right) ; 3.86(\mathrm{~s}, 2 \mathrm{H}, m s-\mathrm{H}) ; 2.33\left(\mathrm{t}, 4 \mathrm{H},{ }^{1} J=6.9 \mathrm{~Hz}\right.$, $\left.\mathrm{CH}_{2}-\mathrm{Hc}\right) ; 2.18\left(\mathrm{~s}, 6 \mathrm{H}, \mathrm{CH}_{3}\right) ; 1.24-1.36(\mathrm{~m}, \mathrm{t} 6+16 \mathrm{H}, J=7.1 \mathrm{~Hz}$, $\left.\mathrm{CH}_{3}-\mathrm{Et}+\mathrm{CH}_{2}-\mathrm{Hc}\right) ; 0.88\left(\mathrm{t}, 6 \mathrm{H},{ }^{1} J=6.9 \mathrm{~Hz}, \mathrm{CH}_{3}-\mathrm{Hc}\right)$.

5,5'-Diethoxycarbonyl-3,3'-di-n-heptyl-4,4'-dimethyldipyrromethane $(10, R=H e p)$. Yield: $58.6 \%$. M.p.: $130-133{ }^{\circ} \mathrm{C} .{ }^{1} \mathrm{H}$ NMR $\left(\mathrm{CCl}_{4}\right) \delta_{\mathrm{H}}$ ppm: 9.81 (bs, 2H, NH); 4.17 (q, 4H, J=7.1 Hz, $\mathrm{OCH}_{2}-$ Et); 3.83 (s, 2H, $m s-\mathrm{H}) ; 2.34$ (t, 4H, $\left.{ }^{1} J=7.2 \mathrm{~Hz}, \mathrm{CH}_{2}-\mathrm{Hp}\right) ; 2.18$ (s, $\left.6 \mathrm{H}, \mathrm{CH}_{3}\right) ; 1.20-1.38$ (m, t 6+20H, J=7.1 Hz, $\mathrm{CH}_{3}-\mathrm{Et}+\mathrm{CH}_{2}-\mathrm{Hp}$ ); $0.88\left(\mathrm{t}, 6 \mathrm{H},{ }^{1} \mathrm{~J}=7.2 \mathrm{~Hz}, \mathrm{CH}_{3}-\mathrm{Hp}\right)$.

5,5'-Diethoxycarbonyl-3,3'-dibenzyl-4,4'-dimethyldipyrromethane $(10, R=B z)$. Yield: $46.8 \%$. M.p.: $121-122{ }^{\circ} \mathrm{C}\left(120{ }^{\circ} \mathrm{C}-\right.$ lit.). ${ }^{[1]]}{ }^{1} \mathrm{H}$ NMR $\left(\mathrm{CCl}_{4}\right) \delta_{\mathrm{H}}$ ppm: 10.05 (bs, $\left.2 \mathrm{H}, \mathrm{NH}\right) ; 7.28-6.82(\mathrm{~m}$, $10 \mathrm{H}, \mathrm{H}-\mathrm{Ph}$ ); 4.03 (q, 4H, J=7.1 Hz, $\left.\mathrm{OCH}_{2}-\mathrm{Et}\right) ; 3.70$ (s, 2H, ms$\left.\mathrm{CH}_{2}\right) ; 3.48$ (s, 4H, $\left.\mathrm{CH}_{2} \mathrm{Ph}\right) ; 1.20$ (t, $6 \mathrm{H}, J=7.1 \mathrm{~Hz}, \mathrm{CH}_{3}-\mathrm{Et}$ ).

2-Carboxy-3-ethyl-4-methyl-5-ethoxycarbonylpyrrole (13, $R=E t)$. 2-Ethoxycarbonyl-3,5-dimethyl-4-ethylpyrrole $(50.0 \mathrm{~g}$, $0.26 \mathrm{~mol}$ ) was dissolved with stirring and cooling in $500 \mathrm{ml}$ of ether, and sulfuryl chloride $(68.5 \mathrm{ml}, 0.85 \mathrm{~mol})$ was gradually added. The mixture was kept overnight at ambient temperature, then ether was distilled off and a mixture of acetone $(100 \mathrm{ml})$ and water $(100 \mathrm{ml})$ was added to the residue. The mixture was refluxed for $20 \mathrm{~min}$, and a solution of sodium acetate trihydrate $(150 \mathrm{~g}, 1.1 \mathrm{~mol})$ in water $(200 \mathrm{ml})$ was added with subsequent reflux for $2 \mathrm{hrs}$. Acetone was distilled off and after cooling the precipitate was filtered and washed with water. The precipitate was then treated with ethanol $(100 \mathrm{ml})$ and $500 \mathrm{ml}$ of aqueous solution of sodium carbonate decahydrate (70.0 g, $0.24 \mathrm{~mol}$ ) was added with stirring. The mixture was then extracted with ether the aqueous solution acidified with diluted $(1: 5 \mathrm{v} / \mathrm{v})$ hydrochloric acid, the sediment was filtered off, washed with water and dried in air at $70^{\circ} \mathrm{C}$. The yield was $41.2 \mathrm{~g}(62.6 \%)$. M.p.: $208-210{ }^{\circ} \mathrm{C} .{ }^{1} \mathrm{H}$ NMR $\left(\mathrm{CDCl}_{3}\right) \delta_{\mathrm{H}}$ ppm: 9.51 (bs, $\left.1 \mathrm{H}, \mathrm{NH}\right)$; 4.39 (q, $2 \mathrm{H}, J=7.1 \mathrm{~Hz}, \mathrm{OCH}_{2}-\mathrm{Et}$ ); 2.82 (q, $2 \mathrm{H},{ }^{1} J=7.5 \mathrm{~Hz}, \mathrm{CH}_{2}-\mathrm{Et}$ ); $2.32\left(\mathrm{~s}, 3 \mathrm{H}, 4-\mathrm{CH}_{3}\right) ; 1.41$ (t, 3H, J=7.1 Hz, $\left.\mathrm{CH}_{3}-\mathrm{OEt}\right) ; 1.15$ (t, $3 \mathrm{H}$, $\left.{ }^{1} J=7.5 \mathrm{~Hz}, \mathrm{CH}_{3}-\mathrm{Et}\right)$.

In a similar way the following compounds were obtained:

2-Carboxy-3-n-butyl-4-methyl-5-ethoxycarbonylpyrrole (13, $R=B u$ ). Yield: $71.0 \%$. M.p.: $178-181{ }^{\circ} \mathrm{C} .{ }^{1} \mathrm{H}$ NMR $\left(\mathrm{CDCl}_{3}\right) \delta_{\mathrm{H}}$ ppm: 9.49 (bs, 1H, NH); 4.39 (q, 2H, $J=7.1 \mathrm{~Hz}, \mathrm{OCH}_{2}-\mathrm{Et}$ ); 2.78 $\left(\mathrm{t}, 2 \mathrm{H},{ }^{1} J=7.5 \mathrm{~Hz}, \mathrm{CH}_{2}-\mathrm{Bu}\right) ; 2.31\left(\mathrm{~s}, 3 \mathrm{H}, 4-\mathrm{CH}_{3}\right) ; 1.51(\mathrm{qv}, 2 \mathrm{H}$, $\left.{ }^{1} J=7.5 \mathrm{~Hz}, \mathrm{CH}_{2}-\mathrm{Bu}\right) ; 1.41\left(\mathrm{t}+1.40 \mathrm{sc}, 5 \mathrm{H}, J=7.1 \mathrm{~Hz},{ }^{1} J=7.5 \mathrm{~Hz}\right.$, $\left.\mathrm{CH}_{3}-\mathrm{OEt}+\mathrm{CH}_{2}-\mathrm{Bu}\right) ; 0.95\left(\mathrm{t}, 3 \mathrm{H},{ }^{1} J=7.5 \mathrm{~Hz}, \mathrm{CH}_{3}-\mathrm{Bu}\right)$. 
2-Iodo-3-ethyl-4-methyl-5-ethoxycarbonylpyrrole $(14, R=E t)$. Potassium bicarbonate $(12.0 \mathrm{~g}, 0.12 \mathrm{~mol})$ in water $(100 \mathrm{ml})$ was added to a solution of 2-carboxy-3-ethyl-4-methyl-5-ethoxycarbonylpyrrole $(9.0 \mathrm{~g}, 0.04 \mathrm{~mol})$ in methanol $(100 \mathrm{ml})$. The mixture was heated to $60^{\circ} \mathrm{C}$, after which a mixture of iodine $(10.5 \mathrm{~g}, 0.04 \mathrm{mmol})$ and potassium iodide $(17.2 \mathrm{~g}, 0.1 \mathrm{~mol})$ in water $(100 \mathrm{ml})$ was gradually added. The mixture was additionally stirred for $15 \mathrm{~min}$, cooled, the precipitate was filtered, washed with water and dried in open air at ambient temperature to constant weight. The yield was 10.6 g (86 \%). M.p.: $113-116^{\circ} \mathrm{C} .{ }^{1} \mathrm{H}$ NMR $\left(\mathrm{CCl}_{4}\right) \delta_{\mathrm{H}}$ ppm: 9.14 (bs, $1 \mathrm{H}$, $\mathrm{NH}) ; 4.37$ (q, $\left.2 \mathrm{H}, J=7.1 \mathrm{~Hz}, \mathrm{OCH}_{2}-\mathrm{Et}\right) ; 2.28\left(\mathrm{t}, 2 \mathrm{H},{ }^{1} J=7.5 \mathrm{~Hz}\right.$, $\left.\mathrm{CH}_{2}-\mathrm{Et}\right) ; 2.30$ (s, $\left.3 \mathrm{H}, \mathrm{CH}_{3}\right) ; 1.27\left(7 \mathrm{H}, J=7.1 \mathrm{~Hz}, \mathrm{CH}_{3}-\mathrm{OEt}\right) ; 1.05$ (t, $\left.3 \mathrm{H},{ }^{1} \mathrm{~J}=7.5 \mathrm{~Hz}, \mathrm{CH}_{3}-\mathrm{Et}\right)$.

2-Iodo-3-n-butyl-4-methyl-5-ethoxycarbonylpyrrole $(14, R=B u)$ was obtained in a similar way. Yield: $77.5 \%$. M.p.: $102-106{ }^{\circ} \mathrm{C}$. ${ }^{1} \mathrm{H}$ NMR $\left(\mathrm{CCl}_{4}\right) \delta_{\mathrm{H}}$ ppm: 9.34 (bs, $\left.1 \mathrm{H}, \mathrm{NH}\right) ; 4.32$ (q, 2H, $J=7.1$ $\left.\mathrm{Hz}, \mathrm{OCH}_{2}-\mathrm{Et}\right) ; 2.33$ (t, $\left.2 \mathrm{H},{ }^{1} \mathrm{~J}=7.2 \mathrm{~Hz}, \mathrm{CH}_{2}-\mathrm{Bu}\right) ; 2.26$ (s, 3H, $\left.\mathrm{CH}_{3}\right)$; 1.31-1.44 (m,t, 7H, J=7.1 Hz, $\left.\mathrm{CH}_{3}-\mathrm{Et}+\mathrm{CH}_{2}-\mathrm{Bu}\right) ; 0.95(\mathrm{t}, 3 \mathrm{H}$, $\left.{ }^{1} J=7.2 \mathrm{~Hz}, \mathrm{CH}_{3}-\mathrm{Bu}\right)$.

3-Ethyl-4-methyl-5-ethoxycarbonylpyrrole $(15, R=E t)$. a) 2-Iodo-3-ethyl-4-methyl-5-ethoxycarbonylpyrrole (15.0 g), tin(II) chloride dihydrate $(12.5 \mathrm{~g})$, potassium iodide $(0.7 \mathrm{~g})$ and concentrated sulfuric acid $(20.0 \mathrm{ml})$ in ethanol $(150 \mathrm{ml})$ were refluxed for $2 \mathrm{hrs}$. The mixture was then poured into an equal volume of water and extracted with ether. The ether was evaporated and the residue distilled under reduced pressure. The yield was $4.3 \mathrm{~g}(48.6 \%)$. b) 2-Carboxy-3-ethyl-4-methyl-5-ethoxycarbonylpyrrole ( $8.0 \mathrm{~g}, 33.8$ $\mathrm{mmol}$ ) was heated on an oil bath at $250{ }^{\circ} \mathrm{C}$ until no more carbon dioxide was evolved ( $2 \mathrm{hrs})$, then the residue was distilled in vacuo of a waterjet pump. The yield was $2.9 \mathrm{~g}(47.4 \%)$. M.p.: $25^{\circ} \mathrm{C} .{ }^{1} \mathrm{H}$ NMR $\left(\mathrm{CCl}_{4}\right) \delta_{\mathrm{H}}$ ppm: 10.03 (bs, $\left.1 \mathrm{H}, \mathrm{NH}\right) ; 6.99(\mathrm{~d}, 1 \mathrm{H}, J=2.9 \mathrm{~Hz}$, $5-\mathrm{H}) ; 4.70$ (q, $\left.2 \mathrm{H},{ }^{1} J=7.2 \mathrm{~Hz}, \mathrm{OCH}_{2}-\mathrm{Et}\right) ; 2.80$ (q, $2 \mathrm{H},{ }^{2} J=7.6 \mathrm{~Hz}$, 4- $\left.\mathrm{CH}_{2}-\mathrm{Et}\right) ; 2.64$ (s, 3H, 3- $\mathrm{CH}_{3}$ ); $1.77^{2}$ (t, 3H, $\left.{ }^{1} J=7.2 \mathrm{~Hz}, \mathrm{CH}_{3}-\mathrm{OEt}\right)$; $1.56\left(\mathrm{t}, 3 \mathrm{H},{ }^{2} \mathrm{~J}=7.6 \mathrm{~Hz}, 4-\mathrm{CH}_{2}-\mathrm{Et}\right)$.

3-n-Butyl-4-methyl-5-ethoxycarbonylpyrrole $(15, R=B u)$ was obtained similarly. Yield: $50.3 \%$ (yellowish oily liquid). ${ }^{1} \mathrm{H}$ NMR $\left(\mathrm{CCl}_{4}\right) \delta_{\mathrm{H}}$ ppm: 10.14 (bs, 1H, NH); $6.95(\mathrm{~d}, 1 \mathrm{H}, J=2.6 \mathrm{~Hz}, 5-\mathrm{H})$; 4.65 (q, $\left.2 \mathrm{H},{ }^{1} \mathrm{~J}=7.1 \mathrm{~Hz}, \mathrm{OCH}_{2}-\mathrm{Et}\right) ; 2.75\left(\mathrm{t}, 2 \mathrm{H},{ }^{2} \mathrm{~J}=7.6 \mathrm{~Hz}, 4-\mathrm{CH}_{2}-\right.$ $\mathrm{Bu}) ; 2.62\left(\mathrm{~s}, 3 \mathrm{H}, 3-\mathrm{CH}_{3}\right) ; 1.25-1.48\left(\mathrm{~m}, \mathrm{t}, 7 \mathrm{H},{ }^{1} \mathrm{~J}=7.1 \mathrm{~Hz}, \mathrm{CH}_{3}-\right.$ $\left.\mathrm{Et}+\mathrm{CH}_{2}-\mathrm{Bu}\right) ; 0.90\left(\mathrm{t}, 3 \mathrm{H},{ }^{1} J=7.2 \mathrm{~Hz}, \mathrm{CH}_{3}-\mathrm{Bu}\right)$.

5,5'-Diethoxycarbonyl-ms-phenyl-3,3'-diethyl-4,4'-dimethyldipyrromethane $\left(\mathbf{1 6}, R=E t, R^{l}=P h\right)$. The solution of 3-ethyl4-methyl-5-ethoxycarbonylpyrrole $(6.3 \mathrm{~g}, 34.8 \mathrm{mmol})$, benzaldehyde $(1.8 \mathrm{ml}, 17.8 \mathrm{mmol})$ and conc. hydrochloric acid $(5.0 \mathrm{ml})$ in methanol $(50 \mathrm{ml})$ was refluxed for $1 \mathrm{~h}$, then cooled, the precipitate was filtered, washed with methanol and dried. The yield was 5.8 g (74.1\%). M.p.: $116-118{ }^{\circ} \mathrm{C} .{ }^{1} \mathrm{H}$ NMR $\left(\mathrm{CCl}_{4}\right) \delta_{\mathrm{H}}$ ppm: 9.71 (bs, $2 \mathrm{H}, \mathrm{NH}) ; 6.79$ (d, $2 \mathrm{H}, J=8.6 \mathrm{~Hz}, 2.6-\mathrm{H}-\mathrm{Ar}) ; 6.58$ (d, $2 \mathrm{H}, J=8.6 \mathrm{~Hz}$, 3.5-H-Ar); 5.40 (s, 1H, $m s-\mathrm{H}) ; 3.95-4.10(\mathrm{~m}), 3.81-394(\mathrm{~m}, 2 \mathrm{x} 2 \mathrm{H}$, $\mathrm{OCH}_{2}-\mathrm{Et}$ ); 2.38 (q, $4 \mathrm{H},{ }^{1} \mathrm{~J}=7.5 \mathrm{~Hz}, \mathrm{CH}_{2}-\mathrm{Et}$ ); 2.20 (s, 6H, 4,4'- $\mathrm{CH}_{3}$ ); 1.16 (t, $\left.6 \mathrm{H},{ }^{2} \mathrm{~J}=7.1 \mathrm{~Hz}, \mathrm{CH}_{3}-\mathrm{OEt}\right) ; 0.90$ (t, 6H, $\left.{ }^{1} \mathrm{~J}=7.5 \mathrm{~Hz}, \mathrm{CH}_{3}-\mathrm{Et}\right)$.

In a similar way the following compounds were obtained:

5,5'-Diethoxycarbonyl-ms-(4-methoxyphenyl)-3,3'-diethyl4,4'-dimethyldipyrromethane (16, $\left.R=E t, R^{l}=4-M e O P h\right)$. Yield: 87.3 \%. M.p.: $126-128{ }^{\circ} \mathrm{C} .{ }^{1} \mathrm{H}$ NMR $\left(\mathrm{CCl}_{4}\right) \delta_{\mathrm{H}}$ ppm: 9.68 (bs, $\left.2 \mathrm{H}, \mathrm{NH}\right)$; 7.05-7.15 (m, 3H, 3.4.5-H-Ph); 7.02 (d, $2 \mathrm{H}, J=7.5 \mathrm{~Hz}, 2.6-\mathrm{H}-\mathrm{Ph}) ; 5.44$ $(\mathrm{s}, 1 \mathrm{H}, m s-\mathrm{H}) ; 3.64\left(\mathrm{~s}, 3 \mathrm{H}, \mathrm{OCH}_{3}\right) ; 3.92-4.01 \mathrm{~m}, 3.83-3.92(\mathrm{~m}, 2 \mathrm{x} 2 \mathrm{H}$, $\mathrm{OCH}_{2}-\mathrm{Et}$ ); 2.36 (q, 4H, ${ }^{1} \mathrm{~J}=7.4 \mathrm{~Hz}, \mathrm{CH}_{2}-\mathrm{Et}$ ); 2.16 (s, 6H, 4,4'- $\mathrm{CH}_{3}$ ); 1.14 (t, $6 \mathrm{H},{ }^{2} J=7.1 \mathrm{~Hz}, \mathrm{CH}_{3}$-OEt); 0.93 (t, $6 \mathrm{H},{ }^{1} J=7.4 \mathrm{~Hz}, \mathrm{CH}_{3}-\mathrm{Et}$ ).

5,5'-Diethoxycarbonyl-ms-phenyl-3,3'-di-n-butyl-4,4'-dimethyldipyrromethane (16, $\left.R=B u, R^{l}=P h\right)$. Yield: $46.7 \%$. ${ }^{1} \mathrm{H}$ NMR $\left(\mathrm{CCl}_{4}\right) \delta_{\mathrm{H}}$ ppm: 9.55 (bs, 2H, NH); 7.10-7.19 (m, 3H, 3.4.5-H-Ph); 6.98 (d, 2H, $J=7.3 \mathrm{~Hz}, 2.6-\mathrm{H}-\mathrm{Ph}) ; 5.51$ (s, $1 \mathrm{H}, m s-\mathrm{H}) ; 3.93-4.08$ (m, 4H, OCH $-\mathrm{Et}$ ); 2.32 (q, 4H, $\left.{ }^{1} \mathrm{~J}=7.4 \mathrm{~Hz}, \mathrm{CH}_{2}-\mathrm{Bu}\right) ; 2.19$ (s, 6H, $\left.4,4^{\prime}-\mathrm{CH}_{3}\right) ; 1.24-1.33\left(\mathrm{~m}, 8 \mathrm{H}, \mathrm{CH}_{2}-\mathrm{Bu}\right) ; 1.20\left(\mathrm{t}, 6 \mathrm{H},{ }^{2} J=7.2 \mathrm{~Hz}\right.$, $\left.\mathrm{CH}_{3}-\mathrm{OEt}\right) ; 0.86\left(\mathrm{t}, 6 \mathrm{H},{ }^{1} \mathrm{~J}=7.4 \mathrm{~Hz}, \mathrm{CH}_{3}-\mathrm{Bu}\right)$.

5,5'-Diethoxycarbonyl-ms-(4-methoxyphenyl)-3,3'-di-nbutyl-4,4'-dimethyldipyrromethane (16, $\left.R=B u, \quad R^{l}=4-M e O P h\right)$.
Yield: $62 \%$. ${ }^{1} \mathrm{H}$ NMR $\left(\mathrm{CCl}_{4}\right) \delta_{\mathrm{H}} \mathrm{ppm}: 9.65$ (bs, $\left.2 \mathrm{H}, \mathrm{NH}\right) ; 6.82(\mathrm{~d}$, $2 \mathrm{H}, J=8.6 \mathrm{~Hz}, 2.6-\mathrm{H}-\mathrm{Ar}$ ); 6.56 (d, 2H, $J=8.6 \mathrm{~Hz}, 3.5-\mathrm{H}-\mathrm{Ar}$ ); 5.53 (s, $1 \mathrm{H}, m s-\mathrm{H}) ; 3.54$ (s, 3H, $\left.\mathrm{OCH}_{3}\right) ; 3.98-4.10$ (m, 4H, OCH $\left.-\mathrm{Et}\right)$; 2.30 (q, $\left.4 \mathrm{H},{ }^{1} J=7.5 \mathrm{~Hz}, \mathrm{CH}_{2}-\mathrm{Bu}\right) ; 2.17$ (s, $\left.6 \mathrm{H}, 4,4^{\prime}-\mathrm{CH}_{3}\right) ; 1.20-1.31$ $\left(\mathrm{m}, 8 \mathrm{H}, \mathrm{CH}_{2}-\mathrm{Bu}\right) ; 1.20\left(\mathrm{t}, 6 \mathrm{H},{ }^{2} \mathrm{~J}=7.1 \mathrm{~Hz}, \mathrm{CH}_{3}-\mathrm{OEt}\right) ; 0.87$ (t, $6 \mathrm{H}$, $\left.{ }^{1} \mathrm{~J}=7.5 \mathrm{~Hz}, \mathrm{CH}_{3}-\mathrm{Bu}\right)$.

A general procedure for the synthesis of 5,5'-unsubstituted dipyrromethanes $[3(X=H), 11]$. A solution of 5,5'-diethoxycarbonyldipyrromethane $(1.0 \mathrm{~g})$ and potassium hydroxide $(1.0 \mathrm{~g}$, $17.8 \mathrm{mmol}$ ) was refluxed in $15 \mathrm{ml}$ of ethylene glycol. The mixture was cooled, poured into water and the precipitate filtered or extracted with methylene chloride, washed with water and dried in air at ambient temperature or with sodium sulfate and the solvent was distilled off under vacuum and the product was used without further purification.

A general procedure for the synthesis of 5,5'-dicarboxydipyrromethanes $(3, \mathrm{X}=\mathrm{COOH})$. A mixture of 5,5' dicarbethoxydipyrrolylmethane $(1.0 \mathrm{~g})$, potassium hydroxide $(2.0 \mathrm{~g}, 35.6 \mathrm{mmol})$, water $(5 \mathrm{ml})$, and methanol $(20 \mathrm{ml})$ was refluxed for 4 hours. The methanol was distilled off and the residue treated with $200 \mathrm{ml}$ of water, filtered and acidified with dilute $(1: 5 \mathrm{v} / \mathrm{v})$ hydrochloric acid to $\mathrm{pH} \sim 5$. The precipitate was filtered, washed with water and dried in air at ambient temperature.

2,8,12,18-Tetra-n-amyl-3,7,13,17-tetramethylporphyrin $(6, R$ $\left.=A m, R^{l}=H\right)$. A solution 4,4'-dimethyl-3,3'-di- $n$-amyldipyrromethane $(0.52 \mathrm{~g}, 1.65 \mathrm{mmol})$, ethyl orthoformate $(1.0 \mathrm{ml}, 6.01 \mathrm{mmol})$ and trifluoroacetic acid $(0.5 \mathrm{ml}, 6.8 \mathrm{mmol})$ in of chloroform $(50 \mathrm{ml})$ was refluxed for $4 \mathrm{hrs}$ (starting from the second hour a weak stream of air was bubbled through). The mixture was cooled and evaporated at ambient temperature. The residue was dissolved in methylene chloride and chromatographed on aluminum oxide (Brockman grade II) using methylene chloride as the eluent. The first red zone of the porphyrin was collected, the solvent was evaporated and the product precipitated with methanol. The porphyrin was filtered, washed with methanol and dried at $70{ }^{\circ} \mathrm{C}$ in air. The yield was $0.47 \mathrm{~g}(87.5 \%)$. Rf: 0.40 (benzene-hexane, 1:1). UV-Vis $\left(\mathrm{CHCl}_{3}\right) \lambda \mathrm{nm}(\lg \varepsilon)$ : 620 (3.80); 566 (3.89); 533 (4.05); 498 (4.17); 399 (5.25). ${ }^{1} \mathrm{H} \mathrm{NMR}\left(\mathrm{CDCl}_{3}\right) \delta_{\mathrm{H}} \mathrm{ppm:}$ $10.11 \mathrm{~s}, 10.10 \mathrm{~s}(2 \mathrm{x} 2 \mathrm{H}, m s-\mathrm{H}) ; 4.09$ (t, $\left.8 \mathrm{H}, J=7.2 \mathrm{~Hz}, \mathrm{CH}_{2}-\mathrm{Am}\right) ; 3.66$ (s, 12H, 3,7,13,17- $\mathrm{CH}_{3}$ ); 2.33 (qv, $8 \mathrm{H}, J=7.2 \mathrm{~Hz}, \mathrm{CH}_{2}-\mathrm{Am}$ ); 1.74 (qv, $8 \mathrm{H}, J=7.2 \mathrm{~Hz}, \mathrm{CH}_{2}-\mathrm{Am}$ ); 1.55 (sc, $\left.8 \mathrm{H}, J=7.2 \mathrm{~Hz}, \mathrm{CH}_{2}-\mathrm{Am}\right) ; 0.98$ (t, $12 \mathrm{H}, J=7.2 \mathrm{~Hz}, \mathrm{CH}_{3}-\mathrm{Am}$ ); -3.75 (bs, $2 \mathrm{H}, \mathrm{NH}$ ). Mass spectrum (MALDI TOF) $m / z: 646.217[\mathrm{M}]^{+}$, calculated: 647.008 .

In a similar way the following compounds were obtained:

2,8,12,18-Tetra-n-propyl-3,7,13,17-tetramethylporphyrin (6, $R=P r, R^{I}=H$ ). Yield: $52.8 \%$. Rf: 0.45 (benzene-hexane, 1:1). UV-Vis $\left(\mathrm{CHCl}_{3}\right) \lambda \mathrm{nm}(\lg \varepsilon): 621$ (3.74); 568 (3.86); 535 (4.03); 499 (4.15); 400 (5.25). ${ }^{1} \mathrm{H}$ NMR $\left(\mathrm{CDCl}_{3}\right) \delta_{\mathrm{H}} \mathrm{ppm}: 10.13 \mathrm{~s}, 10.10$ (s, $2 \times 2 \mathrm{H}, m s-\mathrm{H}) ; 4.09$ (t, $\left.8 \mathrm{H}, J=7.1 \mathrm{~Hz}, \mathrm{CH}_{2}-\mathrm{Pr}\right) ; 3.67$ (s, 12H, $\left.3,7,13,17-\mathrm{CH}_{3}\right) ; 2.35$ (sc, $\left.8 \mathrm{H}, J=7.1 \mathrm{~Hz}, \mathrm{CH}_{2}-\mathrm{Pr}\right) ; 1.30$ (t, $12 \mathrm{H}$, $J=7.1 \mathrm{~Hz}, \mathrm{CH}_{3}-\mathrm{Pr}$ ); -3.73 (bs, 2H, NH). Mass spectrum (MALDI TOF) $m / z: 534.185[\mathrm{M}]^{+}$, calculated: 534.792 .

2,8,12,18-Tetra-n-butyl-3,7,13,17-tetramethylporphyrin (6, $R=B u, R^{l}=H$ ). Yield: $68.8 \%$. $\mathrm{R}_{\mathrm{f}}: 0.36$ (benzene:hexane, 1:1). UV-Vis $\left(\mathrm{CHCl}_{3}\right) \lambda \mathrm{nm}(\lg \varepsilon): 620$ (3.80); 567 (3.89); 534 (4.05); 499 (4.17); 399 (5.25). ${ }^{1} \mathrm{H}$ NMR $\left(\mathrm{CDCl}_{3}\right) \delta_{\mathrm{H}} \mathrm{ppm}: 10.11 \mathrm{~s}, 10.10$ (s, $2 \times 2 \mathrm{H}, m s-\mathrm{H}) ; 4.10\left(\mathrm{t}, 8 \mathrm{H}, J=7.4 \mathrm{~Hz}, \mathrm{CH}_{2}-\mathrm{Bu}\right) ; 3.66(\mathrm{~s}, 12 \mathrm{H}$, $\left.3,7,13,17-\mathrm{CH}_{3}\right) ; 2.31$ (qv, 8H, $J=7.4 \mathrm{~Hz}, \mathrm{CH}_{2}-\mathrm{Bu}$ ); 1.79 (sc, $8 \mathrm{H}$, $J=7.4 \mathrm{~Hz}, \mathrm{CH}_{2}-\mathrm{Bu}$ ); 1.15 (t, $12 \mathrm{H}, J=7.4 \mathrm{~Hz}, \mathrm{CH}_{3}-\mathrm{Bu}$ ); -3.74 (bs, $2 \mathrm{H}, \mathrm{NH}$ ). Mass spectrum (MALDI TOF) $\mathrm{m} / z: 590.294[\mathrm{M}]^{+}$, calculated: 590.900 .

2,8,12,18-Tetra-n-hexyl-3,7,13,17-tetramethylporphyrin (6, $R=H e x, R^{l}=H$ ). Yield: $52.5 \%$. $\mathrm{R}_{\mathrm{f}}$ : 0.65 (benzene:hexane, 1:1). UV-Vis $\left(\mathrm{CHCl}_{3}\right) \lambda \mathrm{nm}(\lg \varepsilon): 620$ (3.70); 566 (3.85); 534 (4.01); 500 (4.11); 400 (5.27). ${ }^{1} \mathrm{H}$ NMR $\left(\mathrm{CDCl}_{3}\right) \delta_{\mathrm{H}} \mathrm{ppm}: 10.11 \mathrm{~s}, 10.10$ $(\mathrm{s}, 2 \times 2 \mathrm{H}, m s-\mathrm{H}) ; 4.09\left(\mathrm{t}, 8 \mathrm{H}, J=7.4 \mathrm{~Hz}, \mathrm{CH}_{2}-\mathrm{Hex}\right) ; 3.65(\mathrm{~s}, 12 \mathrm{H}$ $3,7,13,17-\mathrm{CH}_{3}$ ); 2.33 (qv, 8H, $J=7.4 \mathrm{~Hz}, \mathrm{CH}_{2}-\mathrm{Hex}$ ); 1.78 (qv, 8H, $J=7.4 \mathrm{~Hz}, \mathrm{CH}_{2}-\mathrm{Hex}$ ); 1.53 (qv, $8 \mathrm{H}, J=7.4 \mathrm{~Hz}, \mathrm{CH}_{2}-\mathrm{Hex}$ ); 1.40 (sc, $\left.8 \mathrm{H}, J=7.4 \mathrm{~Hz}, \mathrm{CH}_{2}-\mathrm{Hex}\right), 0.94$ (t, $\left.12 \mathrm{H}, J=7.4 \mathrm{~Hz}, \mathrm{CH}_{3}-\mathrm{Hex}\right) ;-3.74$ 
(bs, 2H, NH). Mass spectrum (MALDI TOF) $m / z: 702.180[\mathrm{M}]^{+}$, calculated: 703.116 .

2,8,12,18-Tetra-n-heptyl-3,7,13,17-tetramethylporphyrin (6, $R=H e p, R^{I}=H$ ). Yield: $43.5 \%$. $\mathrm{R}_{\mathrm{f}}$ : 0.61 (benzene:hexane, 1:1). UV-Vis $\left(\mathrm{CHCl}_{3}\right) \lambda \mathrm{nm}(\lg \varepsilon): 620$ (3.80); 567 (3.89); 533 (4.04); 498 (4.16); 399 (5.19). ${ }^{1} \mathrm{H}$ NMR $\left(\mathrm{CDCl}_{3}\right) \delta_{\mathrm{H}} \mathrm{ppm:} 10.11 \mathrm{~s}, 10.10$ (s, $2 \times 2 \mathrm{H}, m s-\mathrm{H}) ; 4.09\left(\mathrm{t}, 8 \mathrm{H}, J=7.1 \mathrm{~Hz}, \mathrm{CH}_{2}-\mathrm{Hep}\right) ; 3.66(\mathrm{~s}, 12 \mathrm{H}$, $\left.3,7,13,17-\mathrm{CH}_{3}\right) ; 2.32$ (qv, $\left.8 \mathrm{H}, J=7.1 \mathrm{~Hz}, \mathrm{CH}_{2}-\mathrm{Hp}\right) ; 1.76$ (qv, $8 \mathrm{H}$, $\left.J=7.1 \mathrm{~Hz}, \mathrm{CH}_{2}-\mathrm{Hp}\right) ; 1.50-1.62\left(\mathrm{~m}, 8 \mathrm{H}, \mathrm{CH}_{2}-\mathrm{Hp}\right) ; 1.31-1.38(\mathrm{~m}$, $\left.16 \mathrm{H}, \mathrm{CH}_{2}-\mathrm{Hp}\right) 0.89\left(\mathrm{t}, 12 \mathrm{H}, J=7.1 \mathrm{~Hz}, \mathrm{CH}_{3}-\mathrm{Hp}\right) ;-3.73$ (bs, $2 \mathrm{H}$, $\mathrm{NH}$ ). Mass spectrum (MALDI TOF) $\mathrm{m} / \mathrm{z}: 759.180[\mathrm{M}]^{+}$, calculated: 759.224 .

5,15-Diphenyl-3,7,13,17-tetraethyl-2,8,12,18-tetramethylporphyrin (6, $\left.R=E t, R^{l}=P h\right)$. Yield: $35.4 \%$. $\mathrm{R}_{\mathrm{f}}: 0.12$ (benzene:hexane, $1: 1)$. UV-Vis $\left(\mathrm{CHCl}_{3}\right) \lambda \mathrm{nm}(\lg \varepsilon): 627$ (3.45); 576 (392); 543 (3.82); 509 (4.26); 410 (5.32). ${ }^{1} \mathrm{H}$ NMR $\left(\mathrm{CDCl}_{3}\right) \delta_{\mathrm{H}} \mathrm{ppm:} 10.28$ (s, 2H, 10.20-H); 8.21 (d, 4H, J=7.4 Hz, 2.6-H-Ph); 7.83 (t, 2H, $J=7.4 \mathrm{~Hz}, 4-\mathrm{H}-\mathrm{Ph}) ; 7.70$ (t, $4 \mathrm{H}, J=7.4 \mathrm{~Hz}, 3.5-\mathrm{H}-\mathrm{Ph}) ; 3.58$ (s, $12 \mathrm{H}$, $\left.2,8,12,18-\mathrm{CH}_{3}\right) ; 2.81$ (q, 8H, $\left.{ }^{1} J=7.5 \mathrm{~Hz}, \mathrm{CH}_{2}-\mathrm{Et}\right) ; 1.21(\mathrm{t}, 12 \mathrm{H}$, ${ }^{1} J=7.5 \mathrm{~Hz}, 2,8,12,18-\mathrm{CH}_{3}-\mathrm{Et}$ ); -2.15 (bs, $2 \mathrm{H}, \mathrm{NH}$ ). Mass spectrum (MALDI TOF) $\mathrm{m} / \mathrm{z}: 702.180[\mathrm{M}]^{+}$, calculated: 703.116.

5,15-Diphenyl-3, 7,13,17-tetra-n-butyl-2,8,12,18-tetramethylporphyrin (6, $R=B u, R^{I}=P h$ ). Yield: $35.2 \%$. $\mathrm{R}_{\mathrm{f}}: 0.79$ (benzene); 0.40 (benzene:hexane, $1: 1)$. UV-Vis $\left(\mathrm{CHCl}_{3}\right) \lambda \mathrm{nm}(\lg \varepsilon)$ : 625 (3.53); 577 (3.96); 544 (3.87); 510 (4.29); 411 (5.33). ${ }^{1} \mathrm{H}$ NMR $\left(\mathrm{CDCl}_{3}\right)$ $\delta_{\mathrm{H}}$ ppm: $10.28 \mathrm{~s}(2 \mathrm{H}, 10.20-\mathrm{H}) ; 8.21(\mathrm{~d}, 4 \mathrm{H}, J=7.4 \mathrm{~Hz}, 2.6-\mathrm{H}-\mathrm{Ph})$; 7.85 (t, 2H, $J=7.4 \mathrm{~Hz}, 4-\mathrm{H}-\mathrm{Ph}$ ); 7.71 (t, 4H, $J=7.4 \mathrm{~Hz}, 3.5-\mathrm{H}-\mathrm{Ph}$ ); $3.58\left(\mathrm{~s}, 12 \mathrm{H}, 2,8,12,18-\mathrm{CH}_{3}\right) ; 2.71-2.78\left(\mathrm{~m}, 8 \mathrm{H}, \mathrm{CH}_{2}-\mathrm{Bu}\right) ; 1.54-$ $1.64\left(\mathrm{~m}, 8 \mathrm{H}, \mathrm{CH}_{2}-\mathrm{Bu}\right) ; 1.25$ (sc, $\left.8 \mathrm{H},{ }^{1} J=7.3 \mathrm{~Hz}, \mathrm{CH}_{2}-\mathrm{Bu}\right) ; 0.94$ (t, $12 \mathrm{H},{ }^{1} \mathrm{~J}=7.3 \mathrm{~Hz}, \mathrm{C}_{3}-\mathrm{Bu}$ ); $-2.12 \mathrm{bs}$. Mass spectrum (MALDI TOF) $m / z: 743.357[\mathrm{M}]^{+}$, calculated: 743.096 .

5,15-Bis(4-methoxyphenyl)-3,7,13,17-tetra-n-butyl2,8,12,18-tetramethylporphyrin (6, $\left.R=B u, R^{l}=4-M e O P h\right)$. Yield: $39.2 \%$. $\mathrm{R}_{\mathrm{f}}: 0.45$ (benzene). UV-Vis $\left(\mathrm{CHCl}_{3}\right) \lambda \mathrm{nm}(\lg \varepsilon): 628$ (3.32); 578 (3.92); 544 (3.79); 511 (4.29); 412 (5.41). ${ }^{1} \mathrm{H}$ NMR ( $\left.\mathrm{CDCl}_{3}\right)$ $\delta_{\mathrm{H}}$ ppm: $10.24(\mathrm{~s}, 2 \mathrm{H}, 10.20-\mathrm{H}) ; 8.06(\mathrm{~d}, 4 \mathrm{H}, J=8.5 \mathrm{~Hz}, 2.6-\mathrm{H}-$ $\mathrm{Ar}) ; 3.24$ (d, $4 \mathrm{H}, J=8.5 \mathrm{~Hz}, 3.5-\mathrm{H}-\mathrm{Ar}) ; 4.12\left(\mathrm{~s}, 6 \mathrm{H}, \mathrm{CH}_{3} \mathrm{O}\right) ; 3.57$ (s, $\left.12 \mathrm{H}, 2,8,12,18-\mathrm{CH}_{3}\right) ; 2.81\left(\mathrm{t}, 8 \mathrm{H},{ }^{1} J=8.4 \mathrm{~Hz}, \mathrm{CH}_{2}-\mathrm{Bu}\right) ; 1.54-1.63$ (m, $\left.8 \mathrm{H}, \mathrm{CH}_{2}-\mathrm{Bu}\right) ; 1.28\left(\mathrm{sc}, 8 \mathrm{H},{ }^{2} J=7.4 \mathrm{~Hz}, \mathrm{CH}_{2}-\mathrm{Bu}\right) ; 0.93(\mathrm{t}, 12 \mathrm{H}$, ${ }^{2} J=7.4 \mathrm{~Hz}, \mathrm{CH}_{3}-\mathrm{Bu}$ ); -2.14 (bs, $2 \mathrm{H}, \mathrm{NH}$ ). Mass spectrum (MALDI TOF) $m / z: 803.354[\mathrm{M}]^{+}$, calculated: 803.148.

$3,7,13,17$-Tetramethylporphyrin $\left(\mathbf{6}, R, R^{I}=H\right)$. A solution of 4,4'-dimethyldipyrromethane ( $0.55 \mathrm{~g}, 3.16 \mathrm{mmol})$, ethyl orthoformate $(1.0 \mathrm{ml}, 6.01 \mathrm{mmol})$ and trifluoroacetic acid $(0.5 \mathrm{ml}, 6.7$ $\mathrm{mmol}$ ) in chloroform $(50 \mathrm{ml})$ of was refluxed for $4 \mathrm{hrs}$ (from the second the hour a weak stream of air was passed through the mixture). After cooling, diethylamine $(1.0 \mathrm{ml}, 9.6 \mathrm{mmol})$ was added to neutralize the acid and the precipitate was filtered and washed with methylene chloride. The precipitate was then dissolved in methylene chloride with addition of trifluoroacetic acid, the solution was filtered and repricipitation with diethylamine was performed. The precipitate was filtered sediment and washed with methylene chloride. Yield: $30 \mathrm{mg}(5.2 \%)$. UV-Vis $\left(\mathrm{CHCl}_{3}\right) \lambda \mathrm{nm}(\lg \varepsilon)$ : 618 (3.56); 565 (3.78); 527 (3.86); 495 (4.12); 398 (5.27). UV-Vis $\left(\mathrm{CHCl}_{3}-\right.$

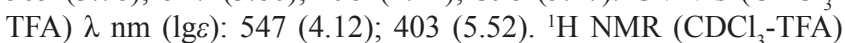
$\delta_{\mathrm{H}}$ ppm: $10.88(\mathrm{~s}, 2 \mathrm{H}, 5.15-\mathrm{H}) ; 10.85(\mathrm{~s}, 2 \mathrm{H}, 10.20-\mathrm{H}) ; 9.55$ (s, 4H, 2,8,12,18-H); -4.09 (bs, $4 \mathrm{H}, \mathrm{NH}$ ). Mass spectrum (MALDI TOF) $m / z: 366.087[\mathrm{M}]^{+}$, calculated: 366.468 .

In a similar way the following compounds were obtained:

2,3,7,8,12,13,17,18-Octamethylporphyrin $\left(6, R=M e, R^{l}=H\right)$. Yield: $31.9 \%$. UV-Vis $\left(\mathrm{CHCl}_{3}\right) \lambda \mathrm{nm}(\lg \varepsilon): 620$ (7.72); 567 (3.82); 532 (3.99); 497 (4.13); 396 (5.19). UV-Vis ( $\left.\mathrm{CHCl}_{3}-\mathrm{TFA}\right) \lambda \mathrm{nm}(\lg \varepsilon)$ : 590 (3.90); 548 (4.23); 401 (5.60). ${ }^{1} \mathrm{H} \mathrm{NMR}\left(\mathrm{CDCl}_{3}-\mathrm{TFA}\right) \delta_{\mathrm{H}} \mathrm{ppm}$ : 10.77 (s, $4 \mathrm{H}, m s-\mathrm{H}) ; 3.70$ (s, 24H, $\mathrm{CH}_{3}$ ); -4.46 (bs, 4H, NH). Mass spectrum (MALDI TOF) m/z: $422.129[\mathrm{M}]^{+}$, calculated: 422.576.
2,8,12,18-Tetraethyl-3,7,13,17-tetramethylporphyrin (6, $R=$ $E t, R^{l}=H$ ). Yield: $65.5 \%$. $\mathrm{R}_{\mathrm{f}}$ (Silufol): 0.22 (benzene:hexane, $1: 1$ ). UV-Vis $\left(\mathrm{CHCl}_{3}\right) \lambda \mathrm{nm}(\lg \varepsilon): 620$ (3.83); 566 (3.91); 532 (4.06); 499 (4.18); 398 (5.21). ${ }^{1} \mathrm{H}$ NMR ( $\mathrm{CDCl}_{3}$-TFA) $\delta_{\mathrm{H}} \mathrm{ppm}$ : $10.71 \mathrm{~s}, 10.70$ (s, $2 \times 2 \mathrm{H}, m s-\mathrm{H}) ; 4.18\left(\mathrm{q}, 8 \mathrm{H}, J=7.1 \mathrm{~Hz}, \mathrm{CH}_{2}-\mathrm{Et}\right) ; 3.70(\mathrm{~s}, 12 \mathrm{H}$, $\mathrm{CH}_{3}$ ); 1.79 (t, $12 \mathrm{H}, J=7.1 \mathrm{~Hz}, \mathrm{CH}_{3}-\mathrm{Et}$ ); -4.01 (bs, 4H, NH). Mass spectrum (MALDI TOF) $\mathrm{m} / \mathrm{z}: 478.158[\mathrm{M}]^{+}$, calculated: 478.684.

2,8,12,18-Tetrabenzyl-3,7,13,17-tetramethylporphyrin $(6, R=$ $\left.B z, R^{l}=H\right)$. Yield: $31.4 \%$. UV-Vis $\left(\mathrm{CHCl}_{3}\right) \lambda \mathrm{nm}(\lg \varepsilon)$ : 623 (3.79); 570 (3.90); 534 (4.05); 500 (4.20); 403 (5.29). ${ }^{1} \mathrm{H}$ NMR ( $\mathrm{CDCl}_{3}$-TFA) $\delta_{\mathrm{H}}$ ppm: $10.71 \mathrm{~s}, 10.50(\mathrm{~s}, 2 \times 2 \mathrm{H}, m s-\mathrm{CH}) ; 7.21-7.32(20 \mathrm{H}, \mathrm{H}-\mathrm{Ph})$; 5.30 (s, 8H, $\left.\mathrm{CH}_{2} \mathrm{Ph}\right) ; 3.65$ (s, $\left.12 \mathrm{H}, \mathrm{CH}_{3}\right) ;-3.69$ (bs, 4H, $\mathrm{NH}$ ). Mass spectrum (MALDI TOF) $\mathrm{m} / z$ : $743.087[\mathrm{M}]^{+}$, calculated: 743.096.

5,15-Bis(4-methoxyphenyl)-3, 7,13,17-tetraethyl-2,8,12,18tetramethylporphyrin (6, $\left.R=E t, R^{l}=4-M e O P h\right)$. Yield: $25.7 \%$. $\mathrm{R}_{\mathrm{f}}: 0.21$ (benzene). UV-Vis $\left(\mathrm{CHCl}_{3}\right) \lambda \mathrm{nm}(\lg \varepsilon): 625$ (3.47); 577 (3.93); 544 (3.80); 510 (4.26); 411 (5.34). ${ }^{1} \mathrm{H}$ NMR ( $\mathrm{CDCl}_{3}$-TFA) $\delta_{\mathrm{H}}$ ppm: 10.07 (s, 2H, 5.10-H); 8.31 (d, $\left.4 \mathrm{H}, J=8.4 \mathrm{~Hz}, 2.6-\mathrm{H}-\mathrm{Ar}\right)$; 7.41 (d, 4H, J=8.4 Hz, 3.5-H-Ar); 4.16 (s, 6H, $\left.\mathrm{OCH}_{3}\right) ; 3.15$ (s, $\left.12 \mathrm{H}, 2,8,12,18-\mathrm{CH}_{3}\right) ; 2.59-2.71\left(\mathrm{~m}, 8 \mathrm{H}, \mathrm{CH}_{2}-\mathrm{Et}\right) ; 0.55(\mathrm{t}, 12 \mathrm{H}$, ${ }^{1} J=7.5 \mathrm{~Hz}, \mathrm{CH}_{3}-\mathrm{Et}$ ); -1.80 (bs, $4 \mathrm{H}, \mathrm{NH}$ ). Mass spectrum (MALDI TOF) $m / z: 690.260[\mathrm{M}]^{+}$, calculated: 692.948 .

Acknowledgements. The current study was performed in accordance with the State Assignment by the Ministry of Education and Science of Russian Federation. The porphyrins were synthesized with the support from the Russian Foundation for Basic Research, grant number 14-23-00204.

\section{References}

1. In: Porphyrins: Spectroscopy, Electrochemistry, Application (Enikolopyan N.S., Ed.), Moscow: Nauka, 1987. 384 p. (in Russ.) [В кн.: Порфирины: спектроскопия, электрохимия, применение (Ениколопян Н.С., ред.), М.: Наука, 1987. 384 c.].

2. Ono N., Kawamura H., Bougauchi M., Maruyama K. Tetrahedron 1990, 46, 7483-7496.

3. Mamardashvili N.G., Semeykin A.S., Golubchikov O.A. Zh. Org. Khim. 1993, 29, 1213-1223 (in Russ.).

4. Golubchikov O.A., Mamardashvili N.Z., Semeikin A.S. Zh. Org. Khim. 1993, 29, 2445-2452 (in Russ.).

5. Berezin D.B., Andrianov V.G., Semeikin A.S. Opt. Spectrosc. 1996, 80, 618-626 (in Russ.).

6. Trofimenko G.M., Semeykin A.S., Berezin M.B., Berezin B.D. Koord. Khim. 1996, 6, 505-509 (in Russ.).

7. Senge M.O., Medforth C.J., Forsyth T.P., Lee D.A., Olmstead M.M., Jentzen W., Pandey R.K., Shelnutt J.A., Smith K.M. Inorg. Chem. 1997, 36, 1149-1163.

8. Berezin M.B., Semeikin A.S., V'yugin A.I., Krestov G.A. Russ. Chem. Bull. 1993, 42, 449-453.

9. Paine III J.B. Synthesis of Pyrroles and of Porphyrins via Single-step Coupling of Dipyrrolic Intermediates. In: The Porphyrins, Ch. 4 (Dolphin D., Ed.) New-York: Academic Press, 1978, pp.100-234.

10. Mironov A.F. In: Porphyrins: Structure, Properties, Synthesis (Enikolopyan N.S., Ed.), Moscow: Nauka, 1985. pp. 282-332 (in Russ.) [Миронов А.Ф. В кн.: Порфириньл: структура, свойства, синтез (Ениколопян Н.С., ред.), М.: Наука, 1985. c. 282-332.].

11. Berezin M.B., Semeikin A.S., V'yugin A.I. Zh. Fiz. Khim. 1996, 70, 1364-1367 (in Russ.). 